Ann. Funct. Anal. 6 (2015), no. 2, 204-211

http://doi.org/10.15352/afa/06-2-17

ISSN: 2008-8752 (electronic)

http://projecteuclid.org/afa

\title{
FUNCTIONAL VERSIONS FOR SOME OPERATOR ENTROPY INEQUALITIES
}

\author{
MUSTAPHA RAÏSSOULI
}

Communicated by J. I. Fujii

\begin{abstract}
In this paper, some functional versions for operator entropies as well as for their related operator inequalities are investigated. The theoretical results obtained by our functional approach immediately imply those of operator version in a simple and nice way.
\end{abstract}

\section{INTRODUCTION}

Let $A$ and $B$ be two positive invertible operators on a Hilbert space $H$ and $\lambda \in(0,1]$. The relative operator entropy $S(A \mid B)$ and the Tsallis operator entropy $T_{\lambda}(A \mid B)$ were defined by, $[2,3,11]$

$$
\begin{gathered}
S(A \mid B)=A^{1 / 2} \log \left(A^{-1 / 2} B A^{-1 / 2}\right) A^{1 / 2}, \\
T_{\lambda}(A \mid B)=\frac{A \sharp_{\lambda} B-A}{\lambda},
\end{gathered}
$$

where

$$
A \sharp_{\lambda} B=A^{1 / 2}\left(A^{-1 / 2} B A^{-1 / 2}\right)^{\lambda} A^{1 / 2}
$$

is the power geometric operator mean of $A$ and $B$. It is well known that $T_{\lambda}(A \mid B)$ extends $S(A \mid B)$ in the sense that the following equality

$$
\lim _{\lambda \downarrow 0} T_{\lambda}(A \mid B)=S(A \mid B)
$$

Date: Received: Jul. 4, 2014; Accepted: Aug. 8, 2014.

2010 Mathematics Subject Classification. Primary 46N10; Secondary 46A20, 47A63, 47N10, 39B62, 52A 41 .

Key words and phrases. Operator inequalities, functional inequalities, operator entropies, convex analysis. 
holds for all positive invertible operators $A$ and $B$. The following operator inequality

$$
A-A B^{-1} A \leq S(A \mid B) \leq B-A
$$

was shown in [2] and later extended for $T_{\lambda}(A \mid B)$ as well, [3]

$$
A-A B^{-1} A \leq T_{\lambda}(A \mid B) \leq B-A
$$

Since then other extensions of $S(A \mid B)$ have been introduced and related operator inequalities have been investigated, see [4] and [7] for instance.

Otherwise, in two earlier papers [8] and [9] we extended $S(A \mid B)$ and $T_{\lambda}(A \mid B)$ from operators to convex functionals, respectively, as follows:

$$
\begin{gathered}
\mathcal{F}(f \mid g)=\int_{0}^{1} \frac{\left((1-t) f^{*}+t g^{*}\right)^{*}-f}{t} d t \\
\mathcal{R}_{\lambda}(f \mid g)=\frac{\mathcal{G}_{\lambda}(f, g)-f}{\lambda}
\end{gathered}
$$

where, for $\lambda \in(0,1)$,

$$
\mathcal{G}_{\lambda}(f, g)=\frac{\sin (\lambda \pi)}{\pi} \int_{0}^{1} \frac{t^{\lambda-1}}{(1-t)^{\lambda}}\left((1-t) f^{*}+t g^{*}\right)^{*} d t
$$

is the power geometric functional mean, extension of $A \sharp_{\lambda} B$ from positive operators to convex functionals. Here, $f^{*}$ denotes the conjugate of $f$ (see Section 2 below).

The following

$$
\mathcal{A}_{\lambda}(f, g):=(1-\lambda) f+\lambda g \text { and } \mathcal{H}_{\lambda}(f, g)=\left((1-\lambda) f^{*}+\lambda g^{*}\right)^{*}
$$

have also been introduced (see [8]) as the extensions of the weighted arithmetic and harmonic means from operators to convex functionals, respectively. The three above functional means satisfy the following double functional inequality

$$
\mathcal{H}_{\lambda}(f, g) \leq \mathcal{G}_{\lambda}(f, g) \leq \mathcal{A}_{\lambda}(f, g)
$$

which extends the weighted arithmetic-geometric-harmonic operator mean inequality, namely

$$
\left((1-\lambda) A^{-1}+\lambda B^{-1}\right)^{-1} \leq A_{\sharp_{\lambda}} B \leq(1-\lambda) A+\lambda B .
$$

The double functional inequality (1.5) allowed us to take

$$
\mathcal{G}_{1}(f, g)=g \quad \text { and } \quad \mathcal{G}_{0}(f, g):=\lim _{\lambda \downarrow 0} \mathcal{G}_{\lambda}(f, g)=f .
$$

For further detail about these functional means, see $[8,9]$ and the related references cited therein.

The previous extensions of $S(A \mid B)$ and $T_{\lambda}(A \mid B)$ from positive operators to convex functionals were investigated in the sense that the following connectionrelationships

$$
\begin{gathered}
\mathcal{F}\left(f_{A} \mid f_{B}\right)=f_{S(A \mid B)}, \\
\mathcal{R}_{\lambda}\left(f_{A} \mid f_{B}\right)=f_{T_{\lambda}(A \mid B)},
\end{gathered}
$$


hold for all positive invertible operators $A$ and $B$, respectively, see [8] and [9]. Here, $f_{A}$ refers to the convex quadratic function generated by the positive operator $A$ i.e. $f_{A}(x)=(1 / 2)\langle A x, x\rangle$ for all $x \in H$.

The fundamental goal of the present paper is to extends the operator inequalities (1.1) and (1.2) for $\mathcal{F}(f \mid g)$ and $\mathcal{R}_{\lambda}(f \mid g)$, respectively. Our functional approach immediately implies, in a nice and fast way, the inequalities (1.1) and (1.2) whose proofs were differently stated in the literature, $[2,3,6]$.

\section{BAsic Notions}

We state here some basic notions needed later. For more details, we refer the reader to $[1,5,10]$ for instance. Let $H$ be a complex Hilbert space with its inner product $\langle.,$.$\rangle and the associate norm \|$.$\| . Let f: H \rightarrow \widetilde{\mathbb{R}}=\mathbb{R} \cup\{\infty\}$ be a functional (in short, we write $f \in \widetilde{\mathbb{R}}^{H}$ ).

- The (extended) space $\widetilde{\mathbb{R}}^{H}$ of such functionals is equipped with the following partial order (so-called the point-wise order)

$$
f, g \in \widetilde{\mathbb{R}}^{H}, \quad f \leq g \Longleftrightarrow f(x) \leq g(x) \text { for all } x \in H,
$$

where we extend the structure of $\mathbb{R}$ to $\mathbb{R} \cup\{-\infty, \infty\}$ by setting

$$
\forall s \in \mathbb{R} \cup\{-\infty, \infty\} \quad-\infty \leq s \leq \infty \text { and } s+\infty=\infty .
$$

Since our involved functionals can take the value $\infty$ then the equality $f-f=0$ does not always hold, since $\infty-\infty=\infty$. For the same reason, the two inequalities $f \leq g$ and $f-g \leq 0$ are not always equivalent.

- The effective domain dom $f$ of $f$ is given by

$$
\operatorname{dom} f=\{x \in H, \quad f(x)<\infty\},
$$

and its conjugate $f^{*}$ is defined through

$$
\forall x^{*} \in H \quad f^{*}\left(x^{*}\right)=\sup _{x \in H}\left\{R e\left\langle x^{*}, x\right\rangle-f(x)\right\}=\sup _{x \in \operatorname{dom} f}\left\{\operatorname{Re}\left\langle x^{*}, x\right\rangle-f(x)\right\} .
$$

As well-known, $f^{* *}:=\left(f^{*}\right)^{*} \leq f, f^{*}$ is always convex lower semi-continuous and, $f \leq g$ implies $g^{*} \leq f^{*}$, for all functionals $f, g \in \widetilde{\mathbb{R}}^{H}$. Further, the conjugate map $f \longmapsto f^{*}$ is point-wise convex in the sense that

$$
\forall f, g \in \widetilde{\mathbb{R}}^{H} \quad \forall t \in(0,1) \quad((1-t) f+t g)^{*} \leq(1-t) f^{*}+t g^{*}
$$

- The sub-differential $\partial f(x)$ of $f$ at $x \in \operatorname{dom} f$ is the (possibly empty) subset of $H$ defined by

$$
x^{*} \in \partial f(x) \Longleftrightarrow \forall y \in H \quad f(y) \geq f(x)+\operatorname{Re}\left\langle y-x, x^{*}\right\rangle,
$$

and it is well-known that

$$
x^{*} \in \partial f(x) \Longleftrightarrow \operatorname{Re}\left\langle x^{*}, x\right\rangle=f^{*}\left(x^{*}\right)+f(x) .
$$

As usual, the symbol $\Gamma_{\circ}(H)$ denotes the cone of all convex lower semi-continuous functions $f \in \widetilde{\mathbb{R}}^{H}$ not identically equal to $\infty$. With this, we have $f^{* *}=f$ if and only if $f \in \Gamma_{\circ}(H)$. Moreover, if $f \in \Gamma_{\circ}(H)$ then, $x^{*} \in \partial f(x)$ if and only if $x \in \partial f^{*}\left(x^{*}\right)$. Denoting by int(dom $f$ ) the topological interior of $\operatorname{dom} f$, we recall 
that if $f \in \Gamma_{\circ}(H)$ and $\operatorname{int}(\operatorname{dom} f)$ is nonempty then, for all $x \in \operatorname{int}(\operatorname{dom} f), f$ is continuous at $x$ and $\partial f(x)$ is nonempty.

- The directional derivative of $f$ at $x$ in the direction $y$ is defined by

$$
f^{\prime}(x, y)=\lim _{t \downarrow 0} \frac{f(x+t y)-f(x)}{t}
$$

provided that this limit exists. In this case, if moreover the map $y \longmapsto f^{\prime}(x, y)$ is linear then we say that $f$ is Gateaux-differentiable (in short G-differentiable) at $x$ and we write $f^{\prime}(x, y)=\nabla f(x)(y)$, where $\nabla f(x)$ is called the (operator) Ggradient of $f$ at $x$. With this, if $f$ is convex and G-differentiable at $x$ then $\partial f(x)=$ $\{\nabla f(x)\}$. It is well-known that every differentiable function is G-differentiable.

The next example will be of interest later.

Example 2.1. Let $A$ and $B$ be as above, $f_{A}$ and $f_{B}$ their generated quadratic functions, respectively. The following assertions hold:

(1) $f_{A} \pm f_{B}=f_{A \pm B}$ and, $f_{A}=(\geq \leq) f_{B}$ if and only if $A=(\geq \leq) B$.

(2) For all $x \in H, f^{*}(x)=(1 / 2)\left\langle A^{-1} x, x\right\rangle$. We can then write $f_{A}^{*}=f_{A^{-1}}$.

(3) Since $A$ is self-adjoint (resp. positive) then $f_{A}$ is continuous (resp. convex). Since $f_{A}$ is quadratic continuous then $f_{A}$ is (infinitely) differentiable and so $f_{A}$ is G-differentiable. To compute explicitly $\partial f_{A}(x)$ we use (2.3) for writing

$$
x^{*} \in \partial f_{A}(x) \Longleftrightarrow \operatorname{Re}\left\langle x^{*}, x\right\rangle=f_{A}(x)+f_{A}^{*}\left(x^{*}\right)=\frac{1}{2}\langle A x, x\rangle+\frac{1}{2}\left\langle A^{-1} x^{*}, x^{*}\right\rangle .
$$

This, with the fact that $A$ is self-adjoint and so $\langle A x, x\rangle=\left\|A^{1 / 2} x\right\|^{2}$, yields

$$
\left\|A^{1 / 2} x\right\|^{2}-2 \operatorname{Re}\left\langle x^{*}, x\right\rangle+\left\|A^{-1 / 2} x^{*}\right\|^{2}=0,
$$

or equivalently $\left\|A^{1 / 2} x-A^{-1 / 2} x^{*}\right\|^{2}=0$. We then deduce $A^{1 / 2} x-A^{-1 / 2} x^{*}=0$ and so $x^{*}=A x$. In summary, $\partial f_{A}(x)=\{A x\}$.

\section{Main Results}

Before stating our first main result, we start with the next needed lemmas.

Lemma 3.1. Let $\lambda \in(0,1)$. Then we have

$$
\int_{0}^{1} \frac{t^{\lambda}}{(1-t)^{\lambda}} d t=\frac{\lambda \pi}{\sin (\lambda \pi)}
$$

Proof. If $\Gamma$ and $B$ denote the standard special functions Gamma and Beta, respectively, then we can write

$$
\int_{0}^{1} \frac{t^{\lambda}}{(1-t)^{\lambda}} d t=B(1+\lambda, 1-\lambda)=\frac{\Gamma(1+\lambda) \Gamma(1-\lambda)}{\Gamma(2)}=\lambda \Gamma(\lambda) \Gamma(1-\lambda)=\frac{\lambda \pi}{\sin (\lambda \pi)} .
$$

Lemma 3.2. [9]. Let $f, g \in \widetilde{\mathbb{R}}^{H}$. Then we have

$$
\lim _{\lambda \downarrow 0} \mathcal{R}_{\lambda}(f \mid g)=\mathcal{F}(f \mid g),
$$

where the limit is taken for the point-wise convergence.

Now, our first main result may be recited as follows. 
Theorem 3.3. Let $f \in \Gamma_{\circ}(H)$ be such that $\operatorname{int}(\operatorname{dom} f)$ is nonempty. Then, for each $\lambda \in(0,1]$, the following inequalities

$$
\begin{aligned}
& \sup _{x^{*} \in \partial f(x)}\left(f^{*}-g^{*}\right)\left(x^{*}\right) \leq \mathcal{R}_{\lambda}(f / g)(x) \leq(g-f)(x) \\
& \sup _{x^{*} \in \partial f(x)}\left(f^{*}-g^{*}\right)\left(x^{*}\right) \leq \mathcal{F}(f / g)(x) \leq(g-f)(x)
\end{aligned}
$$

hold true for all $x \in \operatorname{int}(\operatorname{dom} f)$.

Proof. First, (3.3) follows from (3.2) with Lemma 3.2. We now will show (3.2). From (1.3) and (1.4), with (3.1), it is easy to see that

$$
\mathcal{R}_{\lambda}(f \mid g)=\frac{\sin \lambda \pi}{\lambda \pi} \int_{0}^{1} \frac{t^{\lambda}}{(1-t)^{\lambda}} \frac{\mathcal{H}_{t}(f, g)-f}{t} d t .
$$

Otherwise, (2.2) implies that

$$
\mathcal{H}_{t}(f, g):=\left((1-t) f^{*}+t g^{*}\right)^{*} \leq(1-t) f^{* *}+t g^{* *} \leq(1-t) f+t g,
$$

which, when substituted in (3.4) with a help of (3.1), gives the right inequality of (3.2). We now prove the left inequality of (3.2). By (2.1), for all $x, x^{*} \in H$ we can write

$$
\mathcal{H}_{t}(f, g)(x):=\left((1-t) f^{*}+t g^{*}\right)^{*}(x) \geq \operatorname{Re}\left\langle x, x^{*}\right\rangle-\left((1-t) f^{*}+t g^{*}\right)\left(x^{*}\right) .
$$

If moreover $x \in \operatorname{int}(\operatorname{dom} f) \subset \operatorname{dom} f$ then this latter inequality is equivalent to the next one

$$
\mathcal{H}_{f}(f, g)(x)-f(x) \geq \operatorname{Re}\left\langle x, x^{*}\right\rangle-f^{*}\left(x^{*}\right)-f(x)+t\left(f^{*}-g^{*}\right)\left(x^{*}\right) .
$$

We can take $x^{*} \in \partial f(x)$ since $\partial f(x) \neq \emptyset$ by the assumption, so that according to (2.3) the last inequality becomes

$$
\frac{\mathcal{H}_{t}(f, g)(x)-f(x)}{t} \geq\left(f^{*}-g^{*}\right)\left(x^{*}\right) .
$$

Substituting this in (3.4), with a help of (3.1), we then proved that the next inequality

$$
\mathcal{R}_{\lambda}(f \mid g)(x) \geq\left(f^{*}-g^{*}\right)\left(x^{*}\right)
$$

holds true for all $x \in \operatorname{int}(\operatorname{dom} f)$ and $x^{*} \in \partial f(x)$. It follows that the set

$$
\left\{\left(f^{*}-g^{*}\right)\left(x^{*}\right), x^{*} \in \partial f(x)\right\}
$$

is nonempty and upper bounded, for the point-wise order, by $\mathcal{R}_{\lambda}(f \mid g)(x)$. That is,

$$
\forall x \in \operatorname{int}(\operatorname{dom} f) \quad \mathcal{R}_{\lambda}(f \mid g)(x) \geq \sup _{x^{*} \in \partial f(x)}\left(f^{*}-g^{*}\right)\left(x^{*}\right),
$$

and so the left side of (3.2) is obtained, so completes the proof.

The above theorem has many interesting consequences. First, under a convenient hypothesis on $f$, it can be recited in the following form. 
Corollary 3.4. Let $f \in \Gamma_{\circ}(H)$ be G-differentiable at $x$. Then, for every $\lambda \in$ $(0,1]$, we have

$$
\begin{gathered}
f^{*}(\nabla f(x))-g^{*}(\nabla f(x)) \leq \mathcal{R}_{\lambda}(f / g)(x) \leq(g-f)(x) . \\
f^{*}(\nabla f(x))-g^{*}(\nabla f(x)) \leq \mathcal{F}(f / g)(x) \leq(g-f)(x) .
\end{gathered}
$$

Proof. As already pointed in the above section, since $f \in \Gamma_{\circ}(H)$ and $f$ is Gdifferentiable (then continuous) at $x$ then $\operatorname{int}(\operatorname{dom} f) \neq \emptyset$ and $\partial f(x)=\{\nabla f(x)\}$. Substituting this in the left side of (3.2) and (3.3) we obtain (3.5) and (3.6), respectively.

From the above corollary we find again, under a functional angle, the next known result giving upper and lower bounds of the Tsallis relative operator entropy, [2, 3, 6].

Corollary 3.5. For all positive invertible operators $A$ and $B$ and each $\lambda \in(0,1]$ one has

$$
A-A B^{-1} A \leq T_{\lambda}(A / B) \leq B-A \text { and } A-A B^{-1} A \leq S(A / B) \leq B-A
$$

Proof. Take $f=f_{A}$ and $g=f_{B}$ where $A$ and $B$ are as above. Then, by Example 2.1, $\nabla f(x)=\{A x\}$ for all $x \in H$, and so

$$
f^{*}(\nabla f(x))=f_{A^{-1}}(A x)=f_{A}(x) \text { and } g^{*}(\nabla f(x))=f_{B^{-1}}(A x)=f_{A B^{-1} A}(x) .
$$

Substituting these in (3.5) and (3.6), with (1.6), (1.7) and Example 2.1 again, we obtain the desired operator inequalities.

Now, we state another consequence of the above theorem which, as far as we know, appears to us to be new.

Corollary 3.6. Let $f$ be convex and G-differentiable. Then we have

$$
\inf _{g \in \widetilde{\mathbb{R}}^{H}}\left(g+g^{*} \circ \nabla f\right)=\min _{g \in \widetilde{\mathbb{R}}^{H}}\left(g+g^{*} \circ \nabla f\right)=f+f^{*} \circ \nabla f,
$$

where the "inf" is taken for the point-wise order.

Proof. If $f$ is convex and G-differentiable then (3.5) implies that

$$
\forall x \in H \quad f^{*}(\nabla f(x))-g^{*}(\nabla f(x)) \leq(g-f)(x),
$$

or equivalently

$$
\forall x \in H \quad\left(f+f^{*} \circ \nabla f\right)(x) \leq\left(g+g^{*} \circ \nabla f\right)(x),
$$

from which the desired result follows.

Theorem 3.3 can be formulated in another equivalent version recited in the following remark.

Remark 3.7. With the same hypothesis as in Theorem 3.3, the following inequalities

$$
\begin{gathered}
\left(f^{*}-g^{*}\right)\left(x^{*}\right) \leq \inf _{x \in \partial f^{*}\left(x^{*}\right)} \mathcal{R}_{\lambda}(f \mid g)(x) \leq \inf _{x \in \partial f^{*}\left(x^{*}\right)}(g-f)(x) \\
\left(f^{*}-g^{*}\right)\left(x^{*}\right) \leq \inf _{x \in \partial f^{*}\left(x^{*}\right)} \mathcal{F}(f \mid g)(x) \leq \inf _{x \in \partial f^{*}\left(x^{*}\right)}(g-f)(x)
\end{gathered}
$$


hold for all $x^{*} \in \operatorname{int}\left(\operatorname{dom} f^{*}\right)$.

Finally, we end this section by stating the following example.

Example 3.8. Let $1<p, q<\infty$ be two real numbers and take

$$
\forall x \in H \quad f(x)=\frac{1}{p}\|x\|^{p}, \quad g(x)=\frac{1}{q}\|x\|^{q} .
$$

It is well known that the conjugates of $f$ and $g$ are given by, [1]

$$
\forall x \in H \quad f^{*}(x)=\frac{1}{p^{*}}\|x\|^{p^{*}}, \quad g^{*}(x)=\frac{1}{q^{*}}\|x\|^{q^{*}},
$$

where $1<p^{*}, q^{*}<\infty$ are the conjugates numbers of $p$ and $q$, respectively. Since $f$ is convex and G-differentiable then

$$
\partial f(x)=\{\nabla f(x)\}=\left\{\|x\|^{p-2} x\right\} \text { for all } x \neq 0, \quad \partial f(0)=\{0\} .
$$

Substituting these in the double inequality of Corollary 3.4 we obtain (after all computation and reduction)

$$
\begin{aligned}
\frac{1}{p^{*}}\|\cdot\|^{p}-\frac{1}{q^{*}}\|\cdot\|^{q^{*}(p-1)} & \leq \mathcal{R}_{\lambda}\left(\frac{1}{p}\|\cdot\|^{p} \mid \frac{1}{q}\|\cdot\|^{q}\right) \leq \frac{1}{q}\|\cdot\|^{q}-\frac{1}{p}\|\cdot\|^{p} . \\
\frac{1}{p^{*}}\|\cdot\|^{p}-\frac{1}{q^{*}}\|\cdot\|^{q^{*}(p-1)} & \leq \mathcal{F}\left(\frac{1}{p}\|\cdot\|^{p} \mid \frac{1}{q}\|\cdot\|^{q}\right) \leq \frac{1}{q}\|\cdot\|^{q}-\frac{1}{p}\|\cdot\|^{p} .
\end{aligned}
$$

Acknowledgement. The author would like to thank the anonymous referee for his/her valuable comments and suggestions that have been included in the final version of this manuscript.

\section{REFERENCES}

1. I. Ekeland and R. Temam, Convex Analysis and Variational Problems, Translated from the French. Corrected reprint of the 1976 English edition. Classics in Applied Mathematics, 28. Society for Industrial and Applied Mathematics (SIAM), Philadelphia, PA, 1999.

2. J.I. Fujii and E. Kamei, Relative operator entropy in noncommutative information theory, Math. Japon. 34 (1989), 341-348.

3. S. Furuichi, Inequalities for Tsallis relative entropy and generalized skew information, Linear Multilinear Algebra 59 (2011), no. 10, 1143-1158.

4. T. Furuta, Parameteric extensions of Shannon inequality and its reverse one in Hilbert space operators, Linear Algebra Appl. 381 (2004), 219-235.

5. P.J. Laurent, Approximation et Optimisation, Hermann, 1972.

6. B. Mond, C.E.M. Pearce, J. Pečarić, The logarithmic mean is a mean, Math. Commun. 2 (1997), no. 1, 35-39.

7. M.S. Moslehian, F. Mirzapour and A. Morassaei, Operator entropy inequalities, Colloq. Math. 130 (2013), no. 2, 159-168.

8. M. Raïssouli, Tsallis relative entropy for convex functionals, Int. J. Pure Appl. Math. 51 (2009), no. 4, 555-563.

9. M. Raïssouli, Relative functional entropy in convex analysis, J. Appl. Anal. 17 (2011), 231-239.

10. E. Zeidler, Nonlinear Functional Analysis and its Applications III, Springer-Verlag, 1984.

11. L. Zou, Operator inequalities associated with Tsallis relative operator entropy, Math. Ineq. Appl. (to appear). 
Department of Mathematics, Science Faculty, Taibah University, Al Madinah Al Munawwarah, P. O. Box 30097, Zip Code 41477, Saudi Arabia;

Department of Mathematics, Science Faculty, Moulay Ismail University, MekNES, MOROCCO.

E-mail address: raissouli.mustapha@gmail.com 